\title{
Experimental study on entrainment of Stirling engines to an external pacemaker
}

\author{
Kishin Migimatsu ${ }^{1}$, Hisashi Kada ${ }^{1}$, and Isao T. Tokuda ${ }^{1 a)}$ \\ ${ }^{1}$ Department of Mechanical Engineering, Ritsumeikan University, \\ 1-1-1 Noji-higashi, Kusatsu, Shiga 525-8577, Japan \\ a)isao@fc.ritsumei.ac.jp
}

Received October 7, 2016; Revised March 3, 2017; Published July 1, 2017

\begin{abstract}
The Stirling engine, originally development in 1816 by R. Stirling, has been considered as one of the most efficient systems that convert energy resource to electric power using a thermodynamic cycle ideally close to the Carnot cycle. Use of the Stirling engine for realworld problems, however, has been limited because of its relatively low output power. Towards its more practical applicability, simultaneous operation of many individual Stirling engines is indispensable to increase the output power. This paper presents an experimental study of entraining two Stirling engines to an external pacemaker. Our aim is to achieve synchronized oscillations of the Stirling engines without lowering their oscillation frequencies, because both synchrony and high frequencies are important factors to enhance the total output power. Compared to our previous study of directly coupled Stirling engines, it is shown that the output power is significantly improved in the present framework.
\end{abstract}

Key Words: synchronization, Stirling engine, phase oscillator, pacemaker

\section{Introduction}

The Stirling engine, originally development in 1816 by R. Stirling, has been known as one of the most ecological systems that realize an efficient energy transfer [1]. Using an energy resource that may come, e.g., from a residual heat produced in a power plant or in an industrial factory, the engine system regenerates energy. The cyclic rotation is regulated by the internal air, which is compressed and expanded by level difference in temperature. In theory, the thermodynamic cycle can be designed to be close to the Carnot cycle that minimizes the energy loss. So far, relatively small-size systems have been mainly developed for the Stirling engines that generate low-power electricity. Large-scale engines, on the other hand, have not been widely produced, because of the difficulty of realizing an efficient thermodynamic cycle in a large volume system with a low-friction mechanics. Towards generation of a large electric energy, a population of small-scale Stirling engines can be collected to sum up the individual outputs. To increase the total output power, synchronized operation of the individual engines is indispensable to avoid an energy loss. In our previous study, two Stirling engines were directly coupled to each other so as to realize the synchrony. It has been shown that the synchronized engines produced much higher energy than that of nonsynchronized engines [2]. The disadvantage of the coupled systems was that oscillation frequencies of the coupled engines were 
lowered from their natural frequencies. Since the electric output power is proportional to the square of the oscillation frequency, it is crucial to maintain the level of frequency when the engines are coupled. To overcome this problem, alternative way of synchronization, which does not speed down the engines, should be realized. For this purpose, the present paper introduces an external pacemaker that entrains the Stirling engines. As the pacemaker, it is desired to reuse vibrating sources in natural or artificial environment such as machine vibrations in a production plant, oscillating surface of an automobile road etc. Reusing such vibrations, however, may require a large-scale experimental facility, which does not fit to our basic study. Therefore, as an initial step towards recycling the vibrating resources, we utilize a mechanical metronome as the pacemaker.

In order for an ecological operation of the synchronized engines, it is desired that the engines be entrained to the pacemaker with a small amount of forcing strength. Our approach is therefore based on the framework of weakly coupled limit cycle oscillators [3]. According to the theory of phase reduction [3], a weak interaction is sufficient to entrain a limit cycle oscillator, if $n$ times of its natural frequency is very close to $m$ times of the pacemaker's frequency ( $n, m$ : integers). Because of its simplicity, the theory of weakly coupled phase oscillators has been applied to a variety of systems in science and engineering [4-6] including mutually coupled Stirling engines [2]. Here, the idea of phase oscillators is applied to the entrainment of Stirling engine to an external pacemaker, whose natural frequency is set to be close to $n / m$ of the natural frequency of the Stirling engine. We show that two Stirling engines can be entrained to the pacemaker and, as a result, they achieve synchrony without lowering the oscillation frequencies.

The rest of the paper is organized as follows. In Section 2, the concept of synchronization in forced Stirling engines is clarified. Details of the experimental set-up are provided. Phase oscillator model is introduced to elucidate the mechanism of synchronization in the experimental system. Computational procedure to estimate the electric power generation is also provided. In Section 3, experimental results on entrainment and synchronization of the forced Stirling engines are reported. Importance of the high-frequency synchronized dynamics for efficient power generation is demonstrated. The final Section is devoted for conclusions of our experiments and discussions on the future directions of the present study.

\section{Methods}

\subsection{Definition of synchronization}

As a simplified case, we first consider a single Stirling engine forced by a mechanical metronome and define their synchronization. States of the Stirling engine and the metronome at time $t$ are denoted by vectors $x^{(1)}(t)$ and $x^{(2)}(t)$, respectively. Let the functions $x^{(i)}(\cdot)(i=1,2)$ belong to a function space $\chi$. We define some characteristics functional of the processes as a mapping: $C_{t}: \chi \rightarrow \mathcal{C}$, where $\mathcal{C}$ is the set of possible values of $C_{t}$. Then, the Stirling engine and the metronome are said to be in synchronization, if the following relation holds for all $t[7]$ :

$$
n C_{t}\left[x^{(1)}\left(t+\tau_{1}\right)\right]=m C_{t}\left[x^{(2)}\left(t+\tau_{2}\right)\right],
$$

where $n$ and $m$ are synchronization multiplicities and $\tau_{1}, \tau_{2}$ represent constant values (or phase shifts). For periodic processes [8], by using frequency as the characteristics of the process, the synchronization condition can be defined as

$$
n f_{1}=m F,
$$

where $f_{1}$ and $F$ represent frequencies of the Stirling engine and the metronome, respectively. Under the frequency synchronization, the phase difference between the Stirling engine and the metronome is bounded within a constant interval, i.e., $\left|n \phi_{1}(t)-m \Phi(t)\right|<$ const $\left(\phi_{1}(t)\right.$ : phase of the Stirling engine, $\Phi(t)$ : phase of the metronome).

According to the theory of weakly coupled limit cycle oscillators [3,9], if $n$ times natural frequency of the Stirling engine is close to $m$ times natural frequency of the metronome (i.e., $n \tilde{f}_{1} \approx m F$ ), a weak coupling is sufficient to achieve the frequency synchronization between the Stirling engine and 
the metronome. Here, natural frequency of the Stirling engine (i.e., frequency observed under the condition that the engine is not forced by the metronome) is denoted as $\tilde{f}_{1}$. In the present study, we focus on $1: 2$ synchronization $(n=1, m=2)$, under which the Stirling engine exhibits two oscillation cycles during one cycle of the metronome oscillation.

It should be noted that the electric power is enhanced, when the entrained frequency of the Stirling engine $f_{1}$ exceeds its natural frequency $\tilde{f}_{1}$. This is because the output electric power is proportional to the square of the oscillation frequency of the engine as described in detail in Subsection 2.4. The first experiment therefore provides a basic property on entrainment of the Stirling engine to the mechanical metronome.

As a next step, two Stirling engines are coupled commonly to the same metronome. If the two engines are both entrained to the common pacemaker, the engines are indirectly synchronized with each other. Under the synchronized situation, frequencies of the two engines coincide with each other as $f_{1}=f_{2}$ and their phase difference should be bounded as $\left|\phi_{1}(t)-\phi_{2}(t)\right|<$ const, where the subscript of 1 or 2 denotes indices of the two engines.

For the electric power generation with alternating current, synchronized oscillation of the phases of the engines is of significant importance. In the case that the two engines are synchronized, the summation of their output currents simply doubles the amplitude of their total output, thus producing no energy loss. If the two engines are not synchronized, however, summation of their outputs produces beating, whose amplitude varies in time and reduces the output energy. Thus, synchronized motion of the two engines is indispensable. Our purpose is to achieve synchronization of the two Stirling engines with their frequencies higher than the natural ones.

\subsection{Experimental set-up}

Figure 1(a) shows a set-up for the first entrainment experiment. One Stirling engine (K's Home Planning Co. Inc.) was located on a heater (Sakaguchi E. H VOC Corp.). The heater enabled a temperature control of the metal plate, on which the engine was located, with a precision of $1{ }^{\circ} \mathrm{C}$. The Stirling engine was connected to a mechanical metronome (No. 221, Nikko Seiki Co. Inc.) through a thin plastic cord, which was attached to the piston bar of the engine device. When the metronome bar leans to the right side, the thin plastic cord, which is stretched and becomes tense, pulls the piston bar of the Stirling engine (Fig. 1(b)). In this way, the cord provides a coupling between the Stirling engine and the metronome.

Figure 1(c) shows another set-up for the second experiment, under which two Stirling engines (left and right) were entrained to the common metronome (middle). Each engine was connected to the metronome through a thin plastic cord.

The movement of the Stirling engine as well as the mechanical metronome was recorded by a digital video camera (SONY, HDR-CX180, $30 \mathrm{fps}$ ). To quantify the dynamics, the movie data were analyzed. Namely, location of the oscillating point (e.g., moving piston of the engine and vibrating bar of the metronome) was identified and the time series of the corresponding image intensity was extracted as $\left\{s_{1}(t)\right\},\left\{s_{2}(t)\right\},\{S(t)\}$ for the first and the second Stirling engines and the metronome, respectively. The fast Fourier transform was then applied to compute frequencies $f_{1}, f_{2}, F$ of the engines and the metronome. To examine the phase relationship, phases $\phi_{1}(t), \phi_{2}(t), \Phi(t)$ of the two Stirling engines and the metronome were extracted from the corresponding time series $\left(\left\{s_{1}(t)\right\},\left\{s_{2}(t)\right\},\{S(t)\}\right)$ by the Hilbert transform $[10,11]$.

Entrainment of the Stirling engine to the mechanical metronome is influenced by the coupling strength and the frequency mismatch between them. In our experimental framework, the coupling strength was fixed in each session of the experiment. To vary the frequency mismatch between the Stirling engine and the mechanical metronome, frequency of the metronome was regulated by changing the location of the weight attached to the oscillating bar (i.e., change in center of gravity of the bar varies oscillation frequency of the pendulum). Natural frequency of the Stirling engine, on the other hand, was fixed with a constant heating condition. 


\subsection{Phase oscillator model}

In this subsection, a mathematical model is introduced for the Stirling engine forced by the mechanical metronome. The dynamics of the Stirling engine is in principle described by mechanical equation based on the Lagrangian formalism. It has been known, however, that, if the mechanical dynamics gives rise to limit cycle oscillations and the perturbation given by the metronome is relatively weak, the mechanical equation can be reduced to a phase oscillator model as follows [3, 9].

$$
\begin{aligned}
\frac{d \phi_{1}}{d t} & =\tilde{\omega}_{1}+Z\left(\phi_{1}\right) e(\Phi), \\
\frac{d \phi_{2}}{d t} & =\tilde{\omega}_{2}+Z\left(\phi_{2}\right) e(\Phi-\pi), \\
\frac{d \Phi}{d t} & =\Omega .
\end{aligned}
$$

Here, $\phi_{1}$ and $\phi_{2}$ represent phases of the first and the second Stirling engines, $\tilde{\omega}_{1}$ and $\tilde{\omega}_{2}$ represent their corresponding natural angular frequencies $\left(\tilde{\omega}_{1}=2 \pi \tilde{f}_{1}, \tilde{\omega}_{2}=2 \pi \tilde{f}_{2}\right), Z$ is a phase sensitivity function, and $e$ is an input waveform. $\Phi$ and $\Omega$ represent phase and natural frequency of the metronome. For simplicity, it was assumed that the coupling is uni-directional and dynamics of the metronome was not influenced by the Stirling engine. The phase sensitivity function $Z$, which is in general nonlinear and periodic with respect to $2 \pi$, was approximated by a simple harmonic function as $Z(\phi)=a \cdot \sin \phi+c$. A simple sine function without higher-order Fourier terms was used because it provides a standard formula known to be able to capture the essence of synchronization in coupled oscillators [12]. The parameter values were empirically chosen as $a=30$ and $c=10$.

The input function $e$ was constructed as follows. The forcing input was considered on $(e=1)$ when the cord, which connected the Stirling engine to the metronome, was stretched and became tense (Fig. 1(b)). Otherwise, the input was considered off $(e=0)$. According to the video observation of the experiment, the cord was stretched when the metronome bar was in the angle (" $\theta$ " in Fig. 1(b)) from $17.2^{\circ}$ to $45^{\circ}$ (whole movement range of the metronome was between $-45^{\circ}$ and $45^{\circ}$, where the angle of $0^{\circ}$ corresponds to the case when the metronome bar stands vertically). Assuming a uniform rotation, the angle of $\theta=17.2^{\circ}$ corresponds to phase of $\Phi=0.5 \pi \cdot 17.2 / 45=0.6$ and $\Phi=\pi-0.6$. This leads to the input function of $e(\Phi)=1$ (if $0.6 \leq \Phi<\pi-0.6$ ); $e(\Phi)=0$ (otherwise). For the

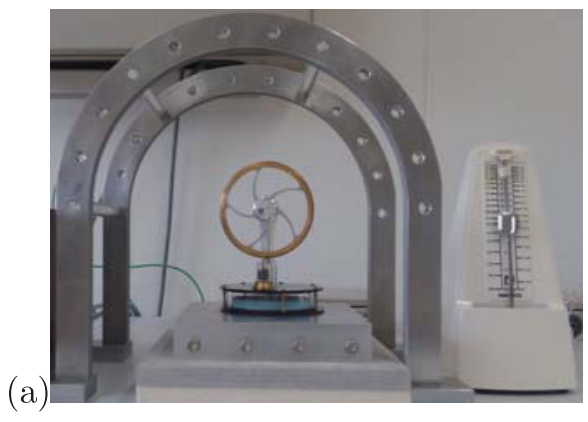

(b)

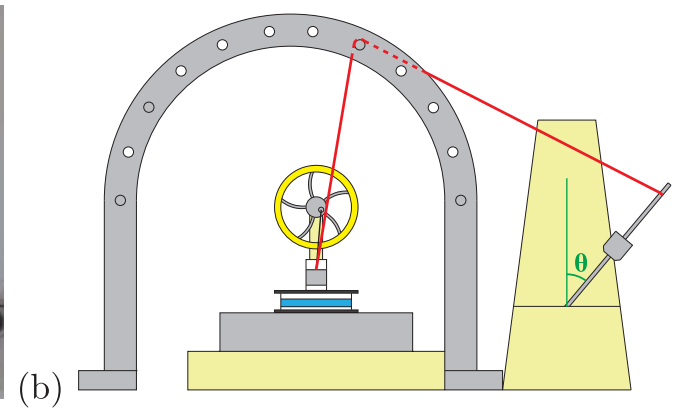

(c)

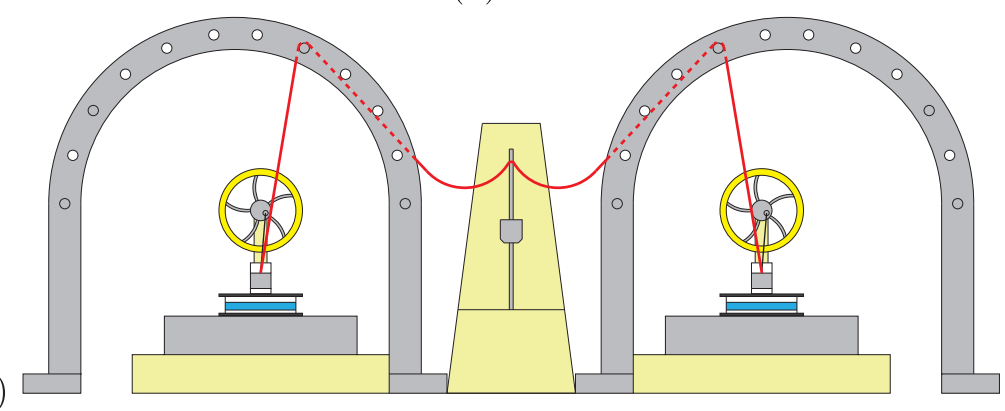

Fig. 1. Experimental set-up. (a) The Stirling engine (middle) is connected to the metronome (right) through thin plastic cord. (b) Schematic illustration of how the plastic cord (red) lifts the piston bar of the Stirling engine in panel (a). Tension of the cord is dependent upon the angle $\theta$ of the metronome bar. (c) Two Stirling engines (left and right) are connected to the metronome (middle) through thin plastic cords. 
Stirling engine, which was located on the opposite side (see Fig. 1(c)), the input was on $(e=1)$ when the metronome bar was in the angle from $-17.2^{\circ}$ to $-45^{\circ}$, leading to the anti-phase input function $e(\Phi-\pi)$ in Eq. (4).

\subsection{Evaluation of output power}

To evaluate an electric power generated from the coupled Stirling engines, we considered an alternator that converts mechanical energy to electric energy in the form of an alternating current [13]. In a stationary magnetic field having a magnetic flux density of $B$, an armature (section area of $S$ ) is supposed to be rotated mechanically by the Stirling engine with its angular frequency $\omega$. For simplicity, resistive load produced from the armature was assumed weak and not considered. The electromotive force $(\mathrm{EMF})$ is then given by

$$
E=B S \omega \sin (\omega t) .
$$

Considering an electric current $I$ that is generated through a resistor $R$ (i.e., $E=I R$ ), the output electric power is evaluated as

$$
P=I E=\frac{E^{2}}{R}=\frac{(B S)^{2}}{R} \omega^{2} \sin ^{2}(\omega t) .
$$

In our framework, the first and the second Stirling engines produce individual electromotive forces as $E_{1}=B S \omega_{1} \sin \left(\omega_{1} t\right)$ and $E_{2}=B S \omega_{2} \sin \left(\omega_{2} t\right)\left(\omega_{1}=2 \pi f_{1}, \omega_{2}=2 \pi f_{2}\right)$, which are serially connected to form a total force. The total force $E_{\text {total }}$ is therefore given by the summation of the left and right forces as $E_{\text {total }}=E_{1}+E_{2}$. This yields the total output power of $P_{\text {total }}=E_{\text {total }}^{2} / R=(B S)^{2}\left\{\omega_{1} \sin \left(\omega_{1} t\right)+\right.$ $\left.\omega_{2} \sin \left(\omega_{2} t\right)\right\}^{2} / R$. Time-average of the total output power is then given by

$$
\left\langle P_{\text {total }}\right\rangle=\lim _{T \rightarrow \infty} \frac{1}{T} \int_{0}^{T} P_{\text {total }} d t .
$$

When the two engines are synchronized, i.e., $\omega_{1}=\omega_{2}=\omega$, the time-averaged total output becomes $\left\langle P_{\text {total }}\right\rangle=2(B S \omega)^{2} / R$. When the two engines are not synchronized $\left(\omega_{1}=\omega, \omega_{2}=\omega+\Delta \omega\right)$, on the other hand, the output becomes $\left\langle P_{\text {total }}\right\rangle \approx(B S \omega)^{2} / R$, which is merely a half of the one obtained under the synchronized condition. This indicates that the synchronized oscillations of the Stirling engines are of great importance for the summation of their output energy. The formulas also show that the output is proportional to the square of the oscillation frequency.

\section{Results}

\subsection{Experiment 1: Entrainment of a single Stirling engine}

As the first experiment, uni-directional coupling, which connects one Stirling engine to the mechanical metronome, was examined. Natural frequency of the Stirling engine was fixed by setting the heater temperature to a constant level, while the natural frequency of the metronome was varied. Figure 2 shows time course of the phase difference between the Stirling engine and the metronome, $\phi_{1}(t)-2 \Phi(t)$. Natural frequency of the Stirling engine, which was measured prior to the entrainment experiment, was $\tilde{f}_{1}=2.05 \mathrm{~Hz}$ for both panels. Natural frequency of the metronome was $F=1.12 \mathrm{~Hz}$ and $F=1.04 \mathrm{~Hz}$ for panels (a) and (b), respectively. Although the phase difference grew linearly in time when the metronome frequency was $1.12 \mathrm{~Hz}$ in (a), it was bounded when the metronome frequency was $1.04 \mathrm{~Hz}$ in (b). This indicates that the Stirling engine was not entrained to the metronome in the case that the frequency mismatch was large $\left(\left|\tilde{f}_{1}-2 F\right|=0.19 \mathrm{~Hz}\right)$. As the frequency mismatch was reduced $\left(\left|\tilde{f}_{1}-2 F\right|=0.03 \mathrm{~Hz}\right)$, the synchronization was achieved.

Figure 3 shows dependence of the oscillation frequency of the Stirling engine (filled circles) on the natural frequency of the metronome. Natural frequency of the Stirling engine (dotted line), which was measured prior to the entrainment experiment, was fixed to $\tilde{f}_{1}=1.86 \mathrm{~Hz}$ in (a), $\tilde{f}_{1}=1.9 \mathrm{~Hz}$ in (b), and $\tilde{f}_{1}=2.05 \mathrm{~Hz}$ in (c). The regime of synchrony is found in the region, where frequency of the Stirling engine lies on a line of $f_{1}=2 F$. The synchronized frequency range for the Stirling engine was $f_{1} \in[1.58,1.9]$ in (a), $f_{1} \in[1.57,1.94]$ in (b), and $f_{1} \in[1.84,2.08]$ in (c). Of particular note 


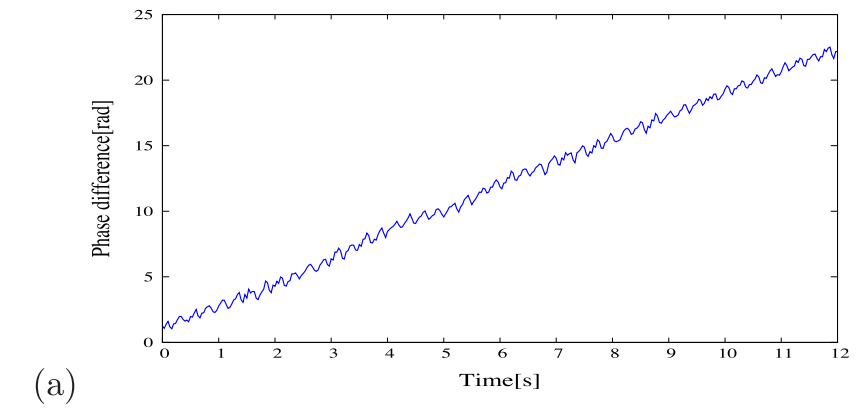

(a)

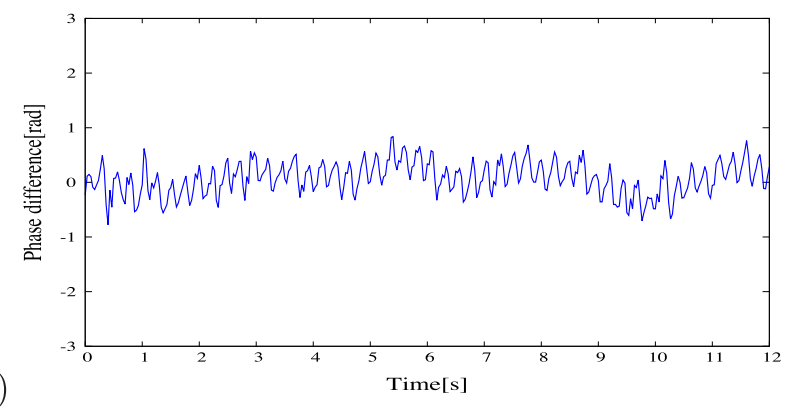

Fig. 2. Time course of phase difference between the Stirling engine and the metronome, $\phi_{1}(t)-2 \Phi(t)$. Natural frequency of the Stirling engine was fixed to $\tilde{f}_{1}=2.05 \mathrm{~Hz}$. Natural frequency of the metronome was set to $F=1.12 \mathrm{~Hz}$ in (a) and $F=1.04 \mathrm{~Hz}$ in (b).

are the points, at which the Stirling engine was entrained to the metronome with a frequency higher than its natural one $\left(f_{1}=1.9 \mathrm{~Hz}\right.$ in Fig. $3(\mathrm{a}), f_{1}=1.94 \mathrm{~Hz}$ in Fig. $3(\mathrm{~b}), f_{1}=2.08 \mathrm{~Hz}$ in Fig. 3(c)). This region is the one desired for an efficient operation of the Stirling engine to generate more output power.

To elucidate the experimental observation, the phase oscillator model of Eqs. (3), (5) was simulated. To realize the experimental situation, natural frequency of the Stirling engine was set to the one observed in the experiment, whereas frequency of the metronome was varied in accordance with the experiment. The simulated curves (red line) are in a good agreement with the experiment (filled circles) in Fig. 3. In the sense that the model reproduced quantitative features of the experimental data quite well, our experiment of the forced Stirling engine is primarily described as a weakly forced limit cycle oscillator.

\subsection{Experiment 2: Synchronization of two Stirling engines coupled to a common metronome}

In the next experiment, two Stirling engines were coupled to the same metronome. Figure 4 shows temporal development of the phase difference between the two Stirling engines, $\phi_{1}(t)-\phi_{2}(t)$. In panel (a), natural frequencies of the two Stirling engines were set to $\tilde{f}_{1}=2.29 \mathrm{~Hz}$ and $\tilde{f}_{2}=2.25 \mathrm{~Hz}$, while natural frequency of the metronome was set to $F=1.18 \mathrm{~Hz}$. The phase difference grew linearly in time, indicating that the two Stirling engines were not synchronized with each other. Difference of the natural frequency between the two Stirling engines was $\left|\tilde{f}_{1}-\tilde{f}_{2}\right|=0.04 \mathrm{~Hz}$, which could not be reduced to zero by the coupling effect. In panel (b), on the other hand, natural frequencies of the two Stirling engines were set to $\tilde{f}_{1}=2.03 \mathrm{~Hz}$ and $\tilde{f}_{2}=2.01 \mathrm{~Hz}$, while natural frequency of the metronome was set to $F=1.065 \mathrm{~Hz}$. Compared to panel (a), the frequency difference was smaller, i.e., $\left|\tilde{f}_{1}-\tilde{f}_{2}\right|=0.02 \mathrm{~Hz}$. In this case, the phase difference was bounded within a finite range, implying that the two Stirling engines were mutually synchronized as the result of their entrainment to the common metronome. It should be noted that the oscillation frequencies of the Stirling engines were both $f_{1}=f_{2}=2.13 \mathrm{~Hz}$, which were higher than the corresponding natural frequencies. The present high-frequency synchrony is desired for the two Stirling engines to generate high output power.

To further examine our observation, the phase model of Eqs. (3), (4), (5) was also simulated. In accordance with the experimental measurement, natural frequencies of the Stirling engines were set to 


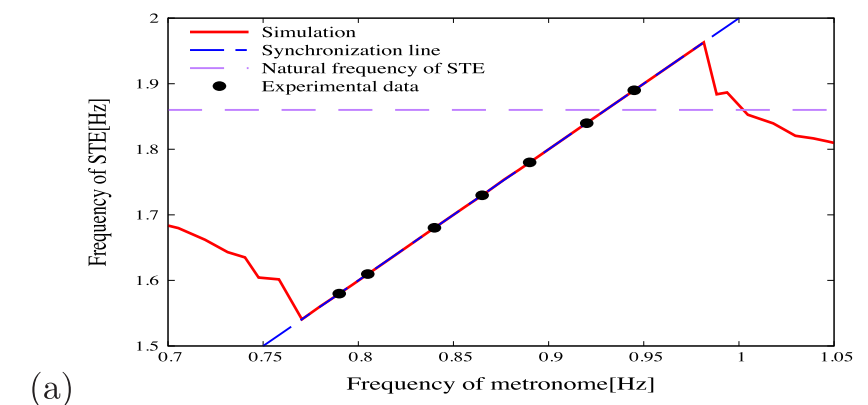

(a)

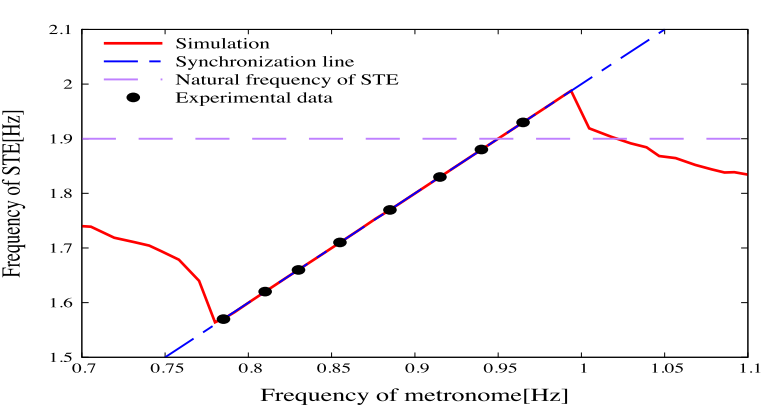

(b)

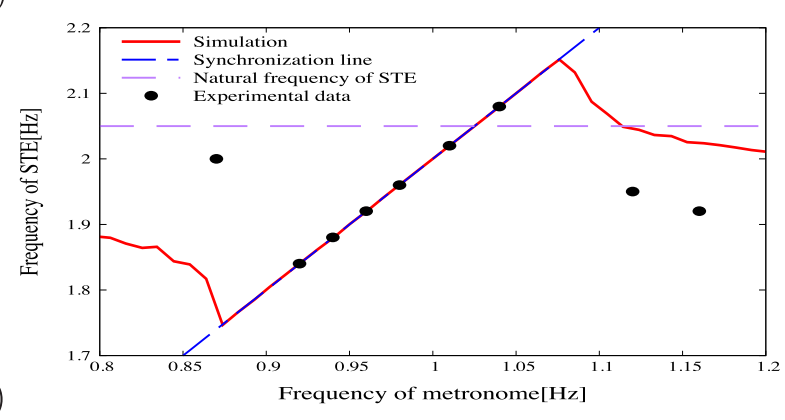

Fig. 3. Dependence of the oscillation frequency of the Stirling engine (filled circles) on the natural frequency of the metronome. Natural frequency of the Stirling engine (purple dotted line) was fixed to $\tilde{f}_{1}=1.86 \mathrm{~Hz}$ in (a), $\tilde{f}_{1}=1.9 \mathrm{~Hz}$ in (b), and $\tilde{f}_{1}=2.05 \mathrm{~Hz}$ in (c). The entrainment condition of $f_{1}=2 F$ is indicated by blue dotted line. The simulation result of the phase model is indicated by red line.

$\tilde{f}_{1}=2.03 \mathrm{~Hz}$ and $\tilde{f}_{2}=2.01 \mathrm{~Hz}$. Interestingly, when the metronome frequency was set to $F=2.13 \mathrm{~Hz}$, which gave rise to synchrony in the experiment, no synchronization was achieved in the simulation. With a slightly varied frequency of $F=2.11 \mathrm{~Hz}$, the two Stirling engines were synchronized (Fig. 4(c)). The reason why the synchronization was not achieved with the experimentally observed frequency of the metronome might be due to a possible effect of the Stirling engine on the dynamics of the metronome. In the phase oscillator model, uni-directional coupling with interaction only from the metronome to the Stirling engine was assumed. To take into account the effect of the Stirling engine on the metronome, bi-directional coupling including interaction from the Stirling engine to the metronome should be also modeled.

Based upon the experimental measurement of the two Stirling engines, the output power was finally estimated according to Eq. (8). For simplicity, the constant product $\frac{(B S)^{2}}{R}$ was normalized as unity. Figure 4(d) shows the total electric power computed for nonsynchronized and synchronized engines. Compared to the power of $\left\langle P_{\text {total }}\right\rangle=161 \mathrm{~W}$ generated by the independent engines with their natural frequencies, the output energy was significantly increased to $\left\langle P_{\text {total }}\right\rangle=358 \mathrm{~W}$ in the synchronized state, indicating the increase of $186 \%$. Compared to the increase of $60 \%$ achieved by the directly coupled Stirling engines in our previous study [2], the present result has been highly improved. This clearly demonstrates the significance of realizing the synchronized oscillations of the Stirling engines for the use of their population sum as the output energy. Since the electric power is proportional to the square of the oscillation frequency (see Subsection 2.4), it is important to maintain the engine frequency high enough. 

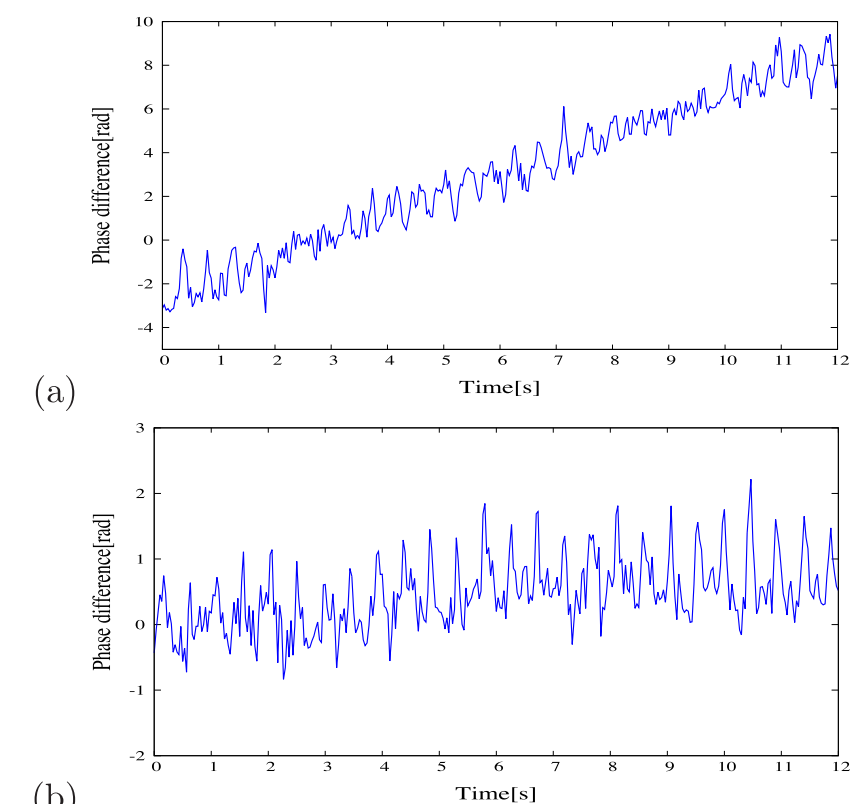

(b)

(c)

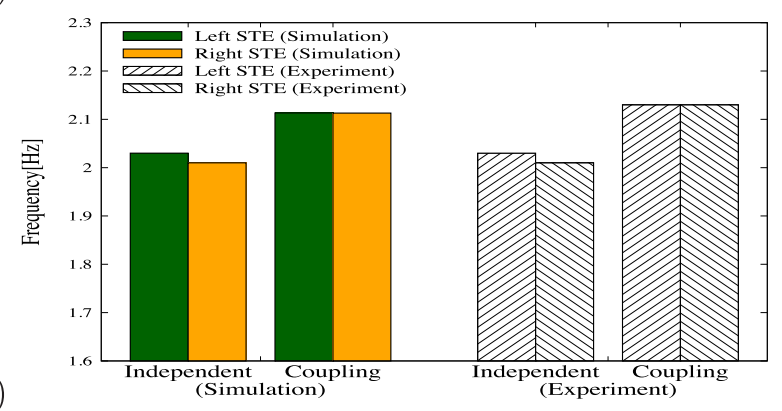

(d)

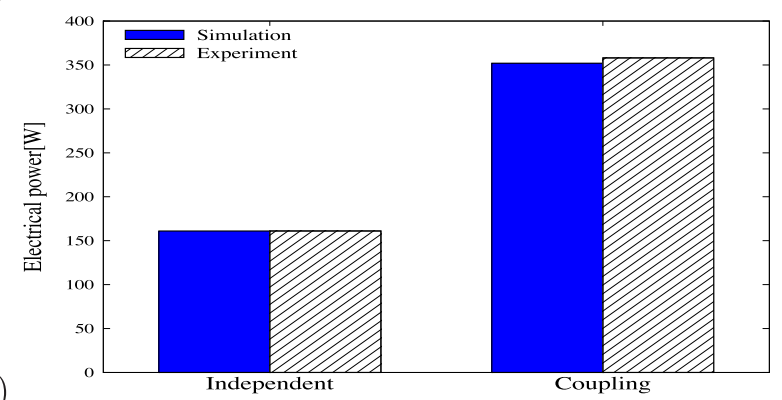

Fig. 4. (a, b) Time course of phase difference between two Stirling engines, $\phi_{1}(t)-\phi_{2}(t)$, connected to the same metronome. In (a), natural frequencies of the two Stirling engines were set to $\tilde{f}_{1}=2.29 \mathrm{~Hz}$ and $\tilde{f}_{2}=2.25 \mathrm{~Hz}$, whereas natural frequency of the metronome was set to $F=1.18 \mathrm{~Hz}$. In (b), natural frequencies of the two Stirling engines were set to $\tilde{f}_{1}=2.03 \mathrm{~Hz}$ and $\tilde{f}_{2}=2.01 \mathrm{~Hz}$, whereas frequency of the metronome was set to $F=1.065 \mathrm{~Hz}$. (c) Oscillation frequencies of the two Stirling engines with and without connection to the metronome. Simulation results are compared with the experiment (experiment: shaded bars, simulation: green and yellow bars). (d) Total electric power generated from the two Stirling engines with and without connection to the metronome. Simulation results are compared with the experiment (experiment: shaded bars, simulation: blue bars).

\section{Discussions}

In the previous study [2], two Stirling engines were directly coupled to each other to realize their synchronization. It has been shown that the synchronized engines produced much higher electric power than that of the nonsynchronized engines. The disadvantage of the directly coupled systems was that oscillation frequencies of the coupled engines were much lowered from their natural frequencies, loosing much of the energy. To overcome this problem, in the present study, mechanical metronome has been introduced as an external pacemaker to entrain dynamics of the Stirling engines. The external 
pacemaker was shown to be crucial for synchronizing the engines by maintaining their oscillation frequencies to a high level.

According to the theory of weakly coupled phase oscillators [3], if $n$ times natural frequency of the Stirling engine is close to $m$ times natural frequency of the metronome, the $\mathrm{n}: \mathrm{m}$ synchronization can be achieved only with a small interaction between them, implying that no strong pacemaker is needed. This is advantageous for an industrial development. Nevertheless, it should be noted that the enhanced oscillations of the Stirling engines realized in the present study come from the oscillation energy of the mechanical metronome. The metronome itself consumes energy for its oscillation, which was not taken into account in the present research. To get rid of the energy resource for the pacemaker, it is desired to reuse vibrating sources from natural or artificial environment. The examples may include machine vibrations in a production plant, oscillating surface of an automobile road or bridge etc. Such pacemakers may not always show periodic oscillations (as the mechanical metronome) but they can often exhibit irregular vibrations. To deal with such irregular pacemakers, noise-induced synchrony [14] shall be considered in our future study. Experiment on synchronization between more than three Stirling engines will be also performed in our forthcoming work.

\section{Acknowledgments}

This study was partially supported by Grants-in-Aid for Scientific Research (No. 16K00343, No. 16K06154, No. 26286086) from Japan Society for the Promotion of Science (JSPS).

\section{References}

[1] G. Walker, Stirling engines, Oxford University Press, New York, 1980.

[2] H. Kada, H. Hojyo, and I.T. Tokuda, "Synchronization of coupled stirling engines," NOLTA, vol. 5, no. 4, pp. 466-475, 2014.

[3] Y. Kuramoto, Chemical oscillations, waves, and turbulence, Springer, Berlin, 1984.

[4] I.Z. Kiss, Y. Zhai, and J.L. Hudson, "Emerging coherence in a population of chemical oscillators," Science, vol. 296, no. 5573, pp. 1676-1678, 2002.

[5] R.F. Galán, G.B. Ermentrout, and N.N. Urban, "Efficient estimation of phase-resetting curves in real neurons and its significance for neural-network modeling," Physical review letters, vol. 94, no. 15 , p. 158101, 2005.

[6] H. Kori and A.S. Mikhailov, "Entrainment of randomly coupled oscillator networks by a pacemaker," Physical review letters, vol. 93, no. 25, p. 254101, 2004.

[7] I.I. Blekhman, A.L. Fradkov, O.P. Tomchina, and D. Bogdanov, "Self-synchronization and controlled synchronization: general definition and example design," Mathematics and Computers in Simulation, vol. 58, no. 4, pp. 367-384, 2002.

[8] C. Hugenii, Horologium oscillatorium, Apud F. Muguet, Parisiis, France, 1673.

[9] A. Pikovsky, M. Rosenblum, and J. Kurths, Synchronization: a universal concept in nonlinear sciences, Cambridge university press, 2003.

[10] D. Gabor, "Theory of communication. part 1: The analysis of information," Electrical Engineers-Part III: Radio and Communication Engineering, Journal of the Institution of, vol. 93, no. 26, pp. 429-441, 1946.

[11] P.F. Panter, Modulation, Noise, and Spectral Analysis: Applied to Information Tranmission, McGraw-Hill Companies, 1965.

[12] J.A. Acebrón, L.L. Bonilla, C.J.P. Vicente, F. Ritort, and R. Spigler, "The kuramoto model: A simple paradigm for synchronization phenomena," Reviews of modern physics, vol. 77, no. 1, p. $137,2005$.

[13] S.P. Thompson, Dynamo-Electricity Machinery: A Manual for Students of Electrotechnics, Cambridge University Press, 2011.

[14] J.N. Teramae and D. Tanaka, "Robustness of the noise-induced phase synchronization in a general class of limit cycle oscillators," Physical Review Letters, vol. 93, no. 20, p. 204103, 2004. 